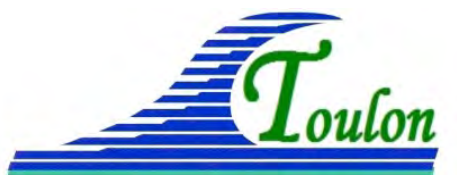

XIV $V^{e ̀ m e s}$ Journées Nationales Génie Côtier - Génie Civil

Toulon, 29 juin au $1^{\text {er }}$ juillet 2016

DOI:10.5150/jngcgc.2016.052 (C) Editions Paralia CFL

disponible en ligne - http://www.paralia.fr - available online

\title{
Un nouvel outil de cartographie des digues
}

\section{Thierry VASSAIL ${ }^{1}$, Maxime FONTAN ${ }^{1}$, Pierre-Eric THEVENIN ${ }^{1}$, Romain BAYLE ${ }^{2}$}

1. OXAND, 49, avenue Franklin Roosevelt, 77210 Avon, France.

thierry.vassail@oxand.com ; maxime.fontan@oxand.com ; pe.thevenin@oxand.com

2. MIAGE, Place Paganon, 38190 Laval, France.

Romain.bayle@miage.eu

\section{Résumé :}

Le territoire français est équipé de plusieurs milliers de kilomètres d'ouvrages dont l'état technique, pour plus de la moitié d'entre eux, n'est pas jugé satisfaisant.

La connaissance de ses ouvrages est pour tout gestionnaire de digues la base de la mise en œuvre d'une politique de gestion cohérente. Il a été montré qu'il n'existait pas à l'heure actuelle d'outil d'analyse à grande échelle, financièrement réaliste, permettant d'identifier de manière quantifiée l'état des digues sur la totalité de leurs linéaires.

Il est proposé une solution nouvelle et économique, pouvant être utilisée pour pallier ce manque technique. La méthodologie envisagée, $\mathrm{SIMEO}^{\mathrm{TM}} 4 \mathrm{D}$, maîtrisée depuis près de 2 décennies dans le domaine des ouvrages d'art et structures de tous types est également adaptée aux digues dont les contrastes de raideur sont pourtant beaucoup plus faibles.

Mots-clés : Digues, Investigation, Cartographie, Mesures, Suivi, Reconnaissance, Optimisation, Simeo ${ }^{\mathrm{TM}} 4 \mathrm{D}$, Fréquences, Caractéristiques dynamiques, Géotechnique.

\section{Les digues en France}

La France métropolitaine, par sa situation géographique favorable, bénéficie d'un réseau hydrographique naturel important et assez bien alimenté en précipitations.

Pour des raisons évidentes de besoins de communications, dans un premier temps, puis énergétique par la suite, la population et les industries se sont ainsi densifiées à proximité des cours d'eau.

La contrepartie de cet avantage naturel est la nécessité de se protéger des excès des variations hydriques de ce réseau. De longue date, la protection contre les inondations a été une préoccupation essentielle. Ainsi, une ordonnance de Charlemagne demandait en 779 de protéger le cours angevin de la Loire par des levées de terre (IGIGABEL et al., 2014). Il existe probablement d'autres références historiques plus anciennes encore. 


\subsection{Inventaire des digues}

Parmi les ouvrages hydrauliques français de régulation ou protection, les digues sont certainement les plus nombreux puisque le linéaire total recensé en 2013 dépassait les 7000 km dont près de 99\% en France métropolitaine.

Ces digues sont classées en 4 catégories d'importance (code de l'environnement art. R.214-113), selon leur hauteur $H$ exprimée en mètres, définie comme la plus grande hauteur mesurée verticalement entre le sommet de l'ouvrage et le terrain naturel du côté de la zone protégée à l'aplomb de ce sommet, et la population maximale $P$ exprimée en nombre d'habitants résidant dans la zone protégée en incluant notamment les populations saisonnières (voir tableaux 1 et 2).

Tableau 1. Classes de digues selon Selon Code de l'environnement art. R.214.113 avant sa modification par le décret 2015-526 du 12 mai 2015.

\begin{tabular}{ll}
\hline Classe de digue & Détermination de la classe \\
\hline $\boldsymbol{A}$ & Digue pour laquelle $H \geq 1$ et $P \geq 50000$ \\
$\boldsymbol{B}$ & Digue non classée en $A$ et pour laquelle $H \geq 1$ et $1000 \leq P \leq 50000$ \\
$\boldsymbol{C}$ & Digue non classée en A ou $B$ et pour laquelle $H \geq 1$ et $10 \leq P \leq 1000$ \\
$\boldsymbol{D}$ & Digue pour laquelle soit $H \leq 1$ et $P \leq 10$ \\
\hline
\end{tabular}

Tableau 2. Recensement 2013 des digues en France (Bilan du SRNH ; MEDDE, 2014).

\begin{tabular}{llllll}
\hline Type & Classe A & Classe B & Classe C & Classe D & Total \\
\hline Linéaire $(\mathrm{km})$ & 340 & 2245 & 3565 & 1482 & 7632 \\
\hline
\end{tabular}

D’autres sources présentent des éléments d'appréciation différents : le CEPRI (2011) par exemple propose un linéaire total de $8598 \mathrm{~km}$ dans son rapport de février 2011 (40\% sont concentrés sur 4 départements : les Bouches du Rhône, le Vaucluse, l'Isère et la Gironde).

Ce linéaire de digues est réparti en plus de 9000 tronçons gérés par plus de 1000 organismes différents. Si la plupart de ces ouvrages sont concédés à des gestionnaires indépendants ou sous la responsabilité de syndicats mixtes clairement identifiés, plus de $1600 \mathrm{~km}$ de digues n'ont pas de gestionnaire reconnu et plusieurs dizaines de tronçons, jugés à enjeux importants, sont dans ce cas.

L'état des ouvrages recensés est assez variable : $3000 \mathrm{~km}$ sont considérés en bon état, plus de $3600 \mathrm{~km}$ apparaissent très dégradés ou présentant des désordres locaux et près de $2000 \mathrm{~km}$ n'avaient pas fait l'objet en 2011 de rendus sur leur état d'endommagement.

Ceci montre bien de manière générale les difficultés de gestion des digues:

i difficulté de recensement des linéaires réels de digues

ii difficulté d'identification du gestionnaire de digues 


\section{XIVìmes Journées Nationales Génie Côtier - Génie Civil \\ Toulon, 29 juin au $1^{\text {er }}$ juillet 2016}

iii difficulté de connaissance de l'état technique réel des corps de digues

Ces éléments doivent de plus être considérés simultanément avec les enjeux en présence.

\subsection{Les enjeux}

Il peut facilement être constaté que cette répartition spatiale des digues se superpose très étroitement avec celle de la population ou de l'activité économique du pays (hors Ile de France).

Les véritables enjeux sont là : dans les zones de protection des digues, nous trouvons d'une part une présence humaine et donc des biens exposés en très grand nombre qui augmentent constamment chaque année et plus rapidement qu'ailleurs dans les zones inondables en France (BOURGUIGNON, 2014) et d'autre part un tissu industriel et tertiaire très dense qui lui aussi augmente, au moins au même rythme que le PIB national.

Les gestionnaires de digues se trouvent ainsi confrontés, directement ou indirectement, au double problème de l'augmentation permanente des enjeux à protéger et à la dégradation inévitable, par simple vieillissement, des éléments de protection nécessaires à cette mise en sécurité du territoire concerné.

Et s'il fallait encore, malheureusement, aggraver ce constat, il faudrait aussi tenir compte des variations potentielles futures de l'aléa météorologique lui-même par les effets du changement climatique (GIEC, 2012). Les experts en charge de l'évaluation du réchauffement de notre climat envisagent en effet de forts changements dans les régimes de précipitations générateurs des crues et submersions. Il n'est pas évident que nos ouvrages de protection soient adaptés à cette évolution.

\section{La gestion pratique des digues}

\subsection{Règlementation en matière de digues}

Le texte concernant la gouvernance de la protection contre les inondations est la loi n²014-58 du 27 janvier 2014 de modernisation de l'action publique territoriale et d'affirmation des métropoles, "loi MAPAM". Elle transfère la compétence de gestion des milieux aquatiques et de prévention des inondations (GEMAPI) aux collectivités territoriales (à certaines communes ou certains établissements publics à fiscalité propre et aux métropoles) qui peuvent en confier la mise en œuvre, en totalité ou partie, à un syndicat mixte, à un établissement public territorial de bassin (EPTB) ou à un établissement public d'aménagement et de gestion de l'eau (EPAGE).

Pour tous les ouvrages dont il a la charge, chaque gestionnaire de digues classées doit respecter certaines obligations qui vont du simple dossier d'ouvrage et au diagnostic de sûreté initial à l'étude de dangers et surtout à la revue de sûreté (digues de classes A et B) réactualisée tous les 10 ans. 
Les ouvrages concédés font également l'objet de circulaires destinées à préciser les modalités des contrôles de la sécurité des ouvrages hydrauliques.

\subsection{La gestion au quotidien}

Quelle que soit l'importance du parc d'ouvrages dont il a la charge, tout gestionnaire de digue doit tout d'abord s'appuyer sur une connaissance physique la plus complète possible de ses ouvrages.

Cette connaissance technique nécessite des équipes multidisciplinaires (hydrauliciens, géotechniciens, forestiers, ...) qui disposent toutes de leurs propres outils spécialisés d'intervention.

L'enjeu technique pour le gestionnaire est de disposer d'un outil adapté à la spécificité d'une digue, sa longueur. Or, aucun des métiers évoqués ci-dessus ne propose réellement d'un tel dispositif à un coût raisonnable. La plupart des investigations classiques étant ponctuelles.

Jusqu'à présent, pour faire face à cette problématique, 2 moyens sont utilisés par tous les gestionnaires :

- L'un est un outil simple mais assez efficace : l'examen visuel ou "walkdown" pour faire un parallèle avec les pratiques dans le domaine industriel,

- L'autre est plutôt un principe de gestion : l'interprétation statistique des données. Il est considéré que la connaissance d'un tronçon en quelques points seulement peut être suffisante pour le décrire dans son intégralité.

Les biais associés à ces 2 principes sont bien connus :

- Pour le premier, il s'agit du traitement subjectif inévitable de l'information liée à la différence d'appréciation entre plusieurs opérateurs,

- Pour le second, il s'agit de la dispersion des caractéristiques physiques du milieu naturel qui rend toute extrapolation à grande échelle assez difficile, voire illusoire.

Au final, le gestionnaire ne dispose pas d'une méthode permettant de rendre compte de l'état général d'une digue avec des critères robustes et reproductibles sans biais.

Pour améliorer ce processus, il pourrait être envisagé, en amont du travail actuel, d'utiliser un outil peu coûteux, rapide à mettre en œuvre et permettant d'obtenir des informations techniques globales sur tout le parc d'ouvrages (quelque-soit la nature de ceux-ci).

En effet, une intervention par paliers de précision croissante permettrait de limiter l'emploi des techniques classiques, précises mais onéreuses, aux seules zones présentant des faiblesses structurales.

\section{Un outil approprié à la gestion des digues}

Disposer d'un outil préalable de cartographie globale serait, pour tout gestionnaire, une source d'efficacité et donc d'économies importantes.

Les gains seraient de 3 ordres : 


\section{XIV èmes Journées Nationales Génie Côtier - Génie Civil \\ Toulon, 29 juin au $1^{\text {er }}$ juillet 2016}

- Economie de moyens d'intervention (coût et rapidité)

- Economie d'utilisation de techniques classiques d'investigation

- Economie de travaux de maintenance

Les opérations classiques de maintenance pourraient ainsi être limitées aux seules zones sensibles repérées en phase amont (figure 1).

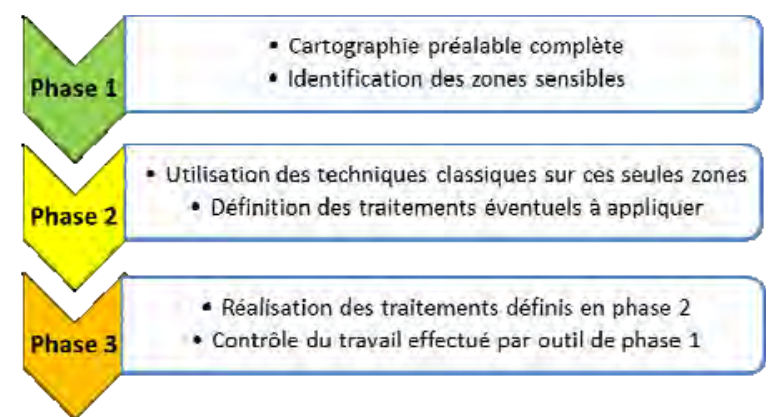

Figure 1. Processus d'intervention proposé.

\subsection{Les méthodes et outils actuels}

Selon les besoins évoqués ci-dessus, 2 principes généraux président à sa définition :

- Rapidité et facilité d'intervention

- Obtention d'informations techniques globales quantifiées et reproductibles

Il faut sans doute ajouter :

- Utilisable en toutes zones et pour tous ouvrages (accès, rives, structures, ...)

Selon ces critères, les techniques habituelles d'investigations semblent disqualifiées, soit parce qu'elles sont coûteuses, soit parce que les techniques sont sensibles à des paramètres perturbateurs (teneur en eau) ou encore parce que leur lenteur de mise en œuvre est inappropriée.

Une analyse simple montre que la technique envisageable doit être peu invasive et que, même si elle est reste très subjective, l'analyse visuelle présente un intérêt par son approche multicritères : les aspects végétation et gestion environnementale peuvent par exemple être ainsi traités.

\subsection{Solution envisageable : exemple d'application}

Parmi toutes les techniques géophysiques utilisables, l'une d'entre elles n'a pas encore, ou peu, été utilisée pour l'analyse des digues : l'analyse des signaux sismiques engendrés par le bruit de fond ambiant. C'est cette technique qu'utilise la méthode SIMEO ${ }^{\mathrm{TM}} 4 \mathrm{D}$ proposée pour l'analyse des digues.

Cette technique est actuellement très couramment employée pour l'analyse des structures. Bien qu'issue de recherches en sismologie et donc relatives aux sols, elle reste actuellement cantonnée aux analyses de type MASW. Celles-ci sont performantes, 


\section{Thème 4 - Ouvrages portuaires, offshore et de plaisance}

mais d'interprétation difficile, et ne permettent pas de traiter rapidement de grands linéaires.

Si l'on sort du contexte géotechnique habituel, une digue peut être considérée comme une structure particulière dont le matériau est de même nature que son support, à la différence d'un ouvrage en béton, par exemple, où le contraste de raideur est très grand entre la structure et le sol de fondation.

Seule la méthode pour l'analyse de la réponse dynamique de l'oscillateur que représente la digue est différente. Elle doit nécessairement être plus fine que pour une structure raide ; mais surtout, la connaissance du milieu environnant (différenciation entre les oscillateurs) doit être parfaitement maîtrisée pour éviter les erreurs d'interprétation.

L'exemple illustré sur la figure 2 montre l'intérêt d'une telle méthode sur un tronçon de digue où une instrumentation de surface a été réalisée en technique légère, par simple pose de capteurs aux sols.

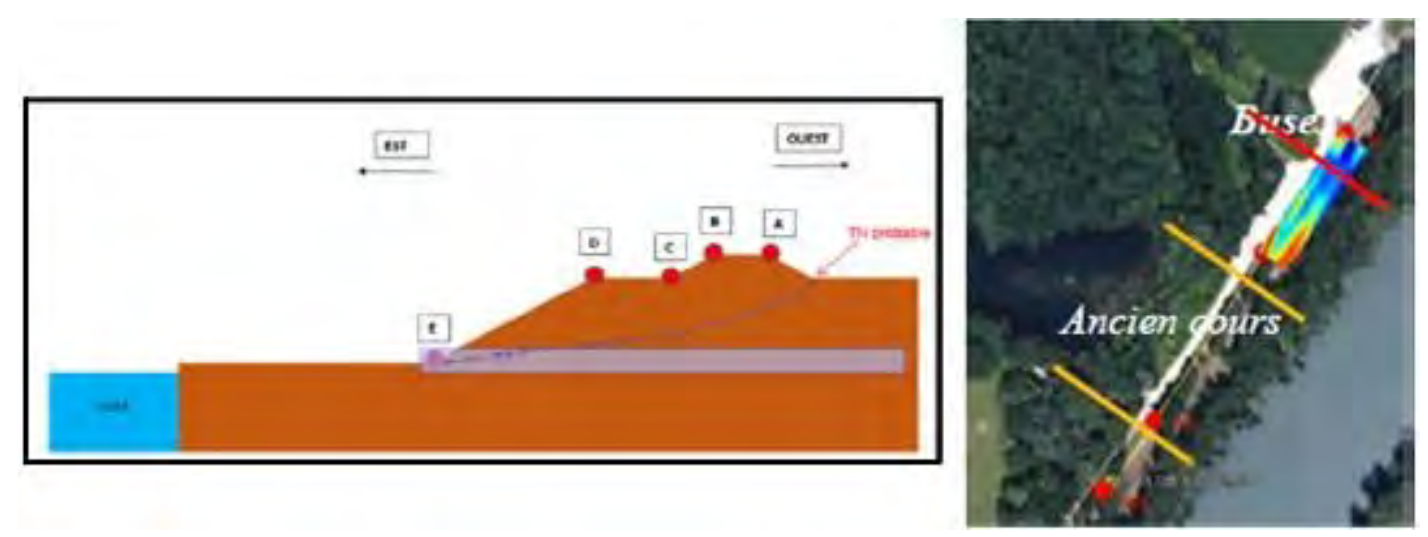

Figure 2. Exemple de digue analysée sous bruit de fond.

L'objectif des mesures était de vérifier les capacités d'identification de singularités physiques dans le corps de digue et dans le sol support. La structure de digue est classique et le matériau constitutif n'est pas différent de celui du support (limons sableux).

Deux types de singularités sont présents sur ce site test. L'une est anthropique : buse métallique de $3 \mathrm{~m}$ de diamètre environ située dans et sous le corps de digue et l'autre est naturelle: ancienne boucle du cours d'eau principal court-circuitée à 2 époques différentes, rectification par comblement du lit mineur puis édification de la digue le long du nouveau tracé.

Les mesures de réponse dynamique (fréquences et amplitudes de modes) du corps de digue ont été réalisées sur la base de profils en travers espacés de $20 \mathrm{~m}$, cette équidistance était choisie, a priori, sans étude préalable. Dans le cadre de cet essai, les mesures ont été réalisées à l'aide de 10 capteurs synchronisés par radio, permettant de ce fait de traiter près de $2000 \mathrm{~m}^{2}$ de digue par heure. 


\section{XIV èmes Journées Nationales Génie Côtier - Génie Civil \\ Toulon, 29 juin au $1^{\text {er }}$ juillet 2016}

La cartographie ci-après est établie sur la base de l'analyse des spectres de réponse en chaque point. L'axe de buse est facilement repérable, le graphe associé est un profil en long des mêmes mesures.

Les figures 3 et 4 traduisent une partie des informations obtenues. Sur la première, il est facilement possible d'observer via une chute fréquentielle les singularités que représentent la buse et l'ancien cours de rivière (au-delà de la cartographie présentée). La deuxième figure permet de juger de l'impact de ces différences sur le corps de digue ; sur la courbe du haut, qui représente la réponse du corps de digue, un pic marqué traduit l'augmentation de "raideur" au niveau de la buse alors que le sol support (courbe du bas) ne connait aucune variation de son état initial. A l'inverse, l'ancienne boucle se traduit par une décompression du sol alors que le corps de digue n'est pas concerné.
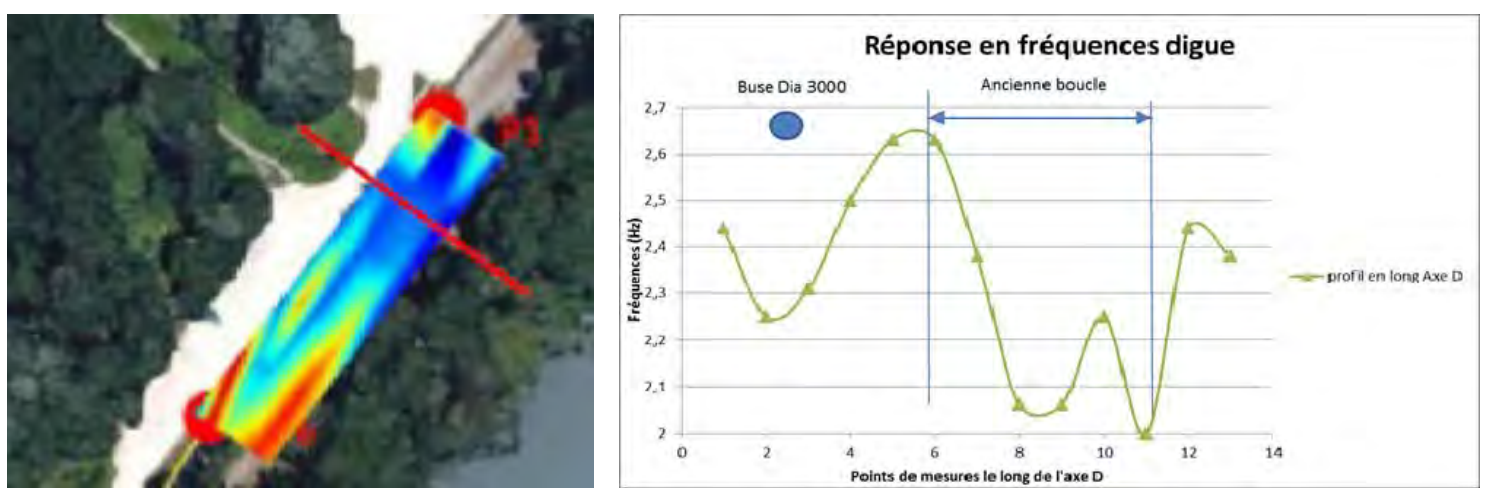

Figure 3. Identification des singularités. La cartographie de gauche ne concerne que la buse métallique.

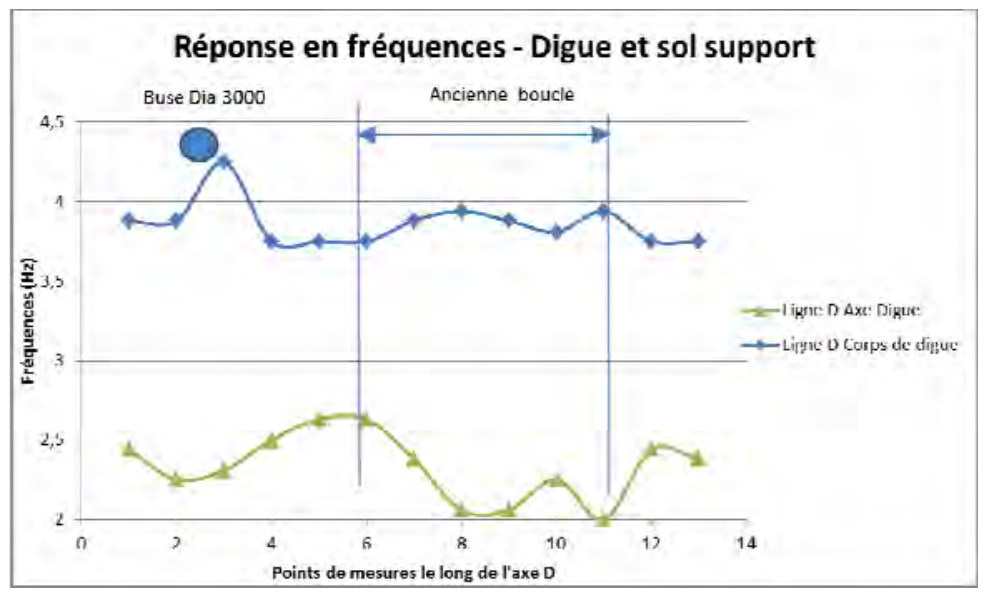

Figure 4. Réponses du corps de digue et du sol support.

Les résultats obtenus sont quantifiés, facilement cartographiables et peuvent être comparés dans le temps quelle que soit la durée entre les prises de mesures.

L'instrumentation ayant permis ces résultats a été réalisée en moins de 2 heures et sans aucun travail de préparation. Cette technique est exploitable en tous lieux, sur de très 
grands linéaires et elle n'est pas dépendante de l'opérateur sur site. Compte tenu des moyens mis en œuvre, elle est particulièrement concurrentielle vis-à-vis des techniques classiques en termes de coût. Il serait ainsi possible de traiter journellement plusieurs kilomètres de digues avec une équipe réduite.

\section{Conclusions}

Le territoire français est équipé de plusieurs milliers de kilomètres d'ouvrages dont l'état technique, pour plus de la moitié d'entre eux n'est pas jugé satisfaisant.

Face à la nécessité de disposer d'un outil de coût raisonnable, pour cartographier de grands linéaires de digues tout en fournissant des données techniques quantifiées, il est proposé une solution nouvelle pouvant être utilisée en toute zone et tout contexte. La technique envisagée, SIMEO ${ }^{\mathrm{TM}} 4 \mathrm{D}$, maîtrisée depuis près de 2 décennies dans le domaine des ouvrages d'art et structures de tous types peut également être adaptée aux digues dont les contrastes de raideur sont beaucoup plus faibles.

\section{Références bibliographiques}

BOURGUIGNON D. (2014). Evènements et territoires - le coût des inondations en France. Analyses spatio-temporelles des dommages assurés. Thèse de doctorat, Université Paul Valéry Montpellier III.

CEPRI -Centre européen des risques et inondations- (2011). La gestion des digues de protection contre les inondations. Rapport du CEPRI, Février 2011. Code de l'environnement, Article R214-113

IGIGABEL M., CHAOUCH V., EL FADILI M. (2014). Coût des protections contre les inondations fluviales. Collection connaissances du CEREMA.

GIEC -Groupe d'experts intergouvernemental sur l'évolution du climat- (2012). Gestion des risques de catastrophes et de phénomènes extrêmes pour les besoins de l'adaptation au changement climatique. Rapport spécial du GIEC.

MEDDE -Ministère de l'écologie, du développement durable et de l'énergie- (2014). Bilan national du contrôle des ouvrages hydrauliques - Année 2013. Rapport du Service des Risques Naturels et Hydrauliques (SRNH). 